\title{
Mechanisms of change: unraveling the roles of modularity and pleiotropy in diversification
}

James J. Lewis ${ }^{1 *}$ and Steven M. Van Belleghem ${ }^{2 *}$

${ }^{1}$ Baker Institute for Animal Health, Cornell University, Ithaca, NY 14853.

${ }^{2}$ Department of Biology, University of Puerto Rico-Rio Piedras, San Juan, PR 00931

Correspondence:

James J. Lewis

jj1336@cornell.edu

Steven M. Van Belleghem

VanBelleghemSteven@hotmail.com

\section{Abstract}

Developmental modularity has long been viewed as a hierarchical organization that facilitates evolution over macro-evolutionary time through modification or co-option of preexisting modules. More recently, developmental modularity has been proposed as a micro-evolutionary mechanism capable of driving rapid evolution of novel color pattern phenotypes between closely related taxa. In this scenario, swapping allelic variants of modular cis-regulatory elements (CREs) via recombination generates novel phenotypes by shuffling preexisting color pattern modules into new arrangements. Recent evidence from Drosophila and butterflies, however, provides a series of examples in which pleiotropic CREs function in multiple developmental contexts. The potential prevalence of pleiotropy in CRE function is a major barrier to the proposed evolutionary role of CRE modules and encourages us to reconsider the relative importance of modularity for microevolutionary change. Here we first review the case for the apparent frequent exchange of modular color pattern phenotypes as a mechanism facilitating diversification. We then contrast this with recent evidence of CRE pleiotropy and argue that exchange of CRE modules should not be the default assumption, even when phenotypes look modular. Finally, we review experimental data on Heliconius butterfly wing patterns - which appear modular - and introduce the concept of evolutionary modularity as an alternative to developmental modularity. Evolutionary modularity reconciles the appearance of modularity in comparative genomic studies of Heliconius color patterns with experimental data supporting a non-modular architecture. We propose that evolutionary modularity provides a potentially important pathway for exchange of phenotypic elements between hybridizing taxa independent of the underlying developmental architecture. 


\section{Introduction}

Diversification of animal coloration has been often used as a model for the genetics and ecology of adaptive evolution. In numerous taxa, adaptive color pattern diversity has repeatedly mapped to relatively few genomic loci. Bird plumage coloration (Campagna et al. 2017; Toews et al. 2016), cryptic hair pigmentation in mice (Steiner, Weber, and Hoekstra 2007; Manceau et al. 2011), and aposematic color patterns in Heliconius butterflies (Reed et al. 2011; Martin et al. 2012; Nadeau et al. 2016; Westerman et al. 2018) are just a few key examples where the loci that differentiate alternate color morphs include only a handful of developmental genes. Consistent with the wellknown trend that gene regulatory mechanisms evolve faster than coding sequence change, many of the loci driving differentiation of adaptive coloration show the strongest signal of divergence between morphs at non-coding loci presumed to capture cis-regulatory variants. Genomic comparisons used to identify color pattern associated regulatory sequence variation repeatedly suggest that combinations of alternative alleles at closely linked loci explain the diversity seen in these color patterns. Consequently, the architecture of this cis-regulation has sometimes been coined as modular and modularity of CREs has been suggested as a potent genetic architecture to explain the rapidly evolving diversity found in these systems (Van Belleghem et al. 2017; Campagna et al. 2017; Wallbank et al. 2016).

Yet despite genome sequence comparisons that favor modular elements as the primary mechanism of phenotypic exchange, there has been little direct evidence to support or reject this model of adaptive evolution. Recent work on the genetic basis of Drosophila morphology and Heliconius butterfly wing color pattern evolution provides the first line of evidence that putative cis-regulatory modules may, in fact, be pleiotropic and non-modular. Here we review the case for modular regulatory elements from genomic datasets and recent findings that suggest we reconsider this model in some scenarios. We then propose a genetic model of hybrid zone differentiation in which the concept of evolutionary modularity can reconcile the apparent modular exchange of alternate alleles with developmental pleiotropy and non-modular genetics.

\section{The case for exchange of modular cis-regulatory elements as a mechanism of diversification}

Modularity has long been a mainstay of developmental biology and evo-devo. Examples of modular genetic mechanisms that underlie trait development, such as melanin patterning across Drosophila species (Rebeiz et al. 2009) and Hox gene expression domains (Kuratani 2009), have demonstrated the importance of modular architectures in determining organismal form. More recently, comparative and functional evolutionary genomics have begun to propose modularity as a mechanism capable of facilitating phenotypic diversification, particularly associated with exchange of modular CREs. In this evolutionary scenario, transfer of autonomous cis-regulatory elements (CREs) via hybridization and recombination of specific genomic loci from one population to another allows for swapping and sorting of discrete phenotypic elements to generate new phenotypes from ancestral genetic components (Wallbank et al. 2016; Van Belleghem et al. 2017). This is most likely to be observed when discrete traits map to one or a few loci of large effect, often referred to as "switch genes". 
The concept of a "module" or "modular element" is critical to grasping the evidence for modularity as a mechanism of evolution. Despite some incongruency in how "modularity" is used, autonomy of function appears to be a common requirement of developmental modules (Wagner, Pavlicev, and Cheverud 2007; Monteiro and Podlaha 2009) and we continue this practice here. For this perspective, we adopt the general definition of developmental modularity as: A genomic locus or set of loci sufficient to autonomously induce a phenotype given any genomic background within a species. This definition has the benefit of being methodologically independent - no specific assays are required for evidence of modularity - and captures the salient requirement that a module must be capable of inducing a phenotype given no other highly derived genetic components.

When considered as a mechanism for producing novel adaptive phenotypes, modular loci seem most likely to underlie variation in phenotypes with discrete pattern elements, such as bird plumage patches or fish scale pigmentation. Specific examples indicative of recombination of modular CREs include capuchino seedeaters in Argentina (Campagna et al. 2017) and North American warblers (Toews et al. 2016) (Figure 1A). In both species groups, discrete plumage color pattern elements appear to be exchanged via hybridization and the strongest signal of genomic differentiation almost exclusively maps to intergenic, putatively cis-regulatory, loci. A modular mechanism of pattern diversification has been proposed in cichlids (Maan and Sefc 2013), and stripe variation among clownfishes (Salis et al. 2018) appears modular as well (Figure 1A). Current direct evidence for the mechanism of phenotypic exchange in fish and birds is sparse and these studies limit speculation on the role of modular loci in generating novel color pattern phenotypes. Nonetheless, closely related, hybridizing taxa that appear to exchange color pattern elements to produce novel phenotypes provide an ideal scenario for evolution via modular genomic loci.

Perhaps the best argument for adaptive evolution via transfer of modular CREs comes from Heliconius hybrid zones in two co-mimetic species. In both Heliconius erato and Heliconius melpomene, regional butterfly populations converge on the same mimicry-related phenotypes to form local morphs with discrete aposematic color pattern elements. In the $H$. melpomene clade, a group of closely related and frequently hybridizing species, some members of the clade have evolved to contain partial phenotypes completely present in other populations, such as the sole presence of rayed hindwing or red forewing triangle patterns in the absence of the other element (Wallbank et al. 2016). This scenario repeats in H. erato, where butterfly morphs again form narrow hybrid zones in which discrete red wing pattern phenotypes appear to be exchanged between morphs (Van Belleghem et al. 2017) (Figure 1A). Importantly, in both species, the exchange of red pattern elements: A) maps back to a cis-regulatory region distal to the "switch" gene optix, and B) associates with recombination of specific genomic haplotypes at these loci that transfer via recombination between neighboring populations with shared wing color pattern elements (Figure 1B).

So where might we expect modular evolution of phenotypes? The case for modularity in comparative genomics is not without limits, and almost certainly requires both hybridization and recombination. Several cases of recurrent morphological adaption have been shown to be driven by convergent evolution due to independent mutations at the same loci, such as the repeated evolution of warning coloration in bumblebees (Tian et al. 2019) and pelvic reduction in stickleback fish (Chan et al. 2010). In both of these cases, the convergent morphology occurs in non-hybridizing populations, suggesting that ecological factors can drive the appearance of shared 
regulatory alleles. Despite this, where recombination can be a driving force in phenotype diversity, modularity is an attractive hypothesis that posits a simple mechanistic process by which new adaptive phenotypes can be rapidly gained or lost.

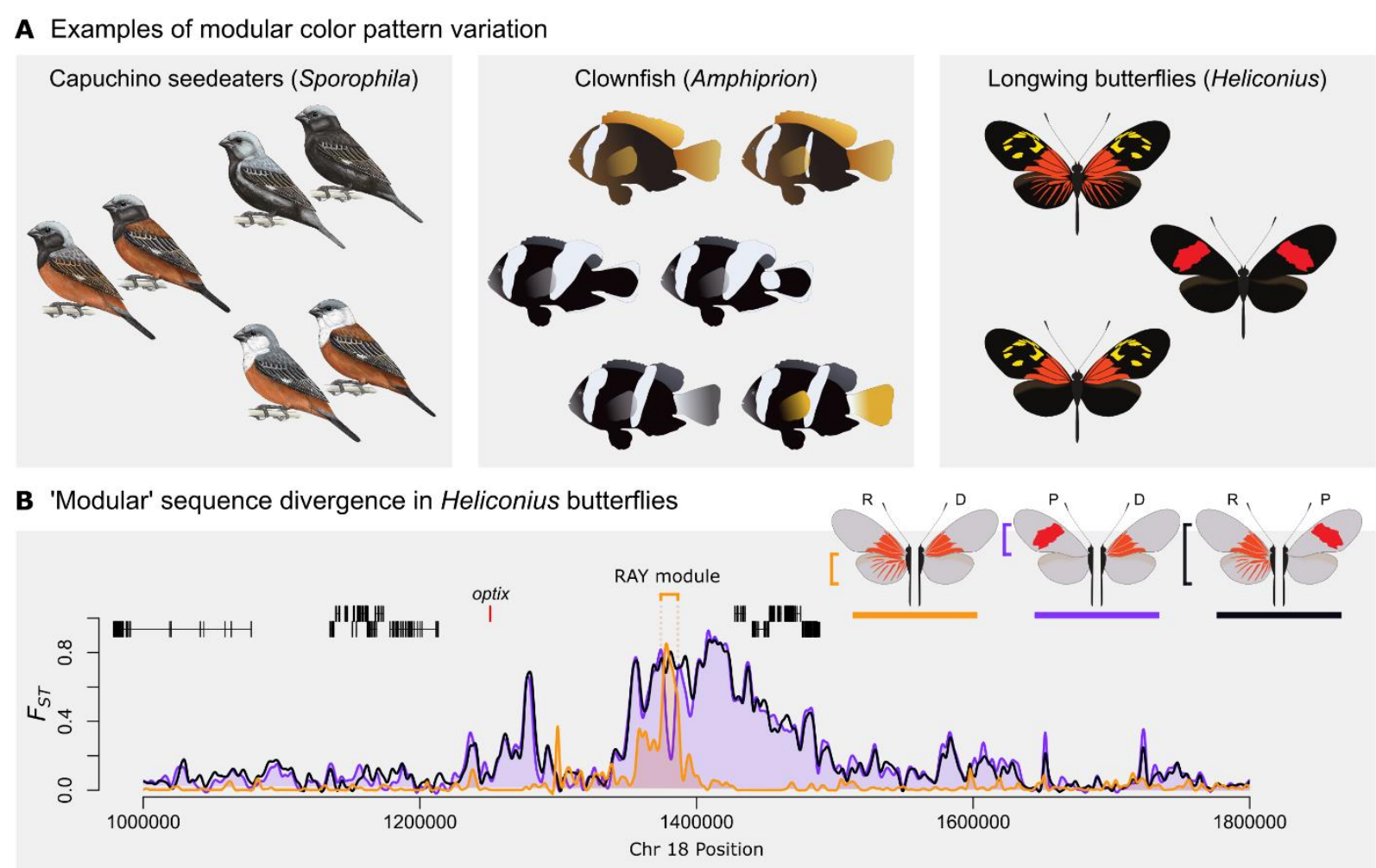

Figure 1. Examples of putative modular color pattern diversity. (A) Examples of potential exchange of modular phenotypes via recombination include plumage patterns in capuchino seedeaters (left, reprinted from Campagna et al. 2017), white stripes in clownfish (middle, modified from Salis et al. 2018), and red wing color patterns in longwing butterflies (right). (B) Non-coding divergence associated with differentiation between red wing pattern morphs in Heliconius. Orange: Differentiation between Radiate (R) and Dennis (D) morphs; Purple: Differentiation between Postman (P) and Dennis (D) morphs; and Black: Differentiation between Radiate (R) and Postman (P) morphs. A single locus shows a putative cis-regulatory module controlling differentiation between the Radiate and Dennis morphs.

\section{CRE pleiotropy and non-modular gene regulation}

While indirect evidence for recombination of modular CREs between geographically close and genetically related taxa is abundant, recent studies in Drosophila, cell lines, and Heliconius butterflies suggest pervasive CRE pleiotropy and interdependence and present several counterexamples to the key principles that support CRE-derived phenotype modularity. This, in turn, requires that we reconsider whether exchange of CRE modules should be the default hypothesis in cases where modular phenotypes appear to be swapped by neighboring populations. Evidence against two principles that favor exchange of CRE modules counter the assumptions that A) CREs are highly tissue-specific and that B) recombination of existing CREs is more important for rapid evolutionary change compared to de novo mutations. We discuss these findings and their consequences for CRE and phenotypic modularity in more detail below. 
First, genes are frequently found to be highly pleiotropic, while cis-regulatory loci are assumed to be highly tissue-specific (Carroll 2008; Prud'homme, Gompel, and Carroll 2007). This principle provides a foundation for exchange of a phenotype-specific CRE without any corresponding alteration of fitness from undesirable pleiotropic effects. In support of this principle, many early examples of trait evolution found that one or two enhancer elements drive variation in phenotypes. Yet recent evidence suggests that cis-regulatory loci may often be substantially more pleiotropic than initially expected. The overall prevalence of enhancer pleiotropy has been well covered by Sabarís et al. (2019), but some aspects of CRE biology that suggest pleiotropic elements may frequently play an important role in generating novel traits are worth considering further.

For example, pioneering work by the ENCODE project found that CRE availability, an accessible chromatin state important for CRE activity, tends to be maintained through cell lineages (Stergachis et al. 2013). Thus, enhancers activated in earlier cell types are often available for use through much of the remainder of development. It is likely then, that these elements could be reutilized or co-opted by evolutionary processes to drive new expression patterns instead of generating a suite of novel CREs de novo (Monteiro and Podlaha 2009). Important for evolution, CREs active in multiple tissues or during extended periods of development show increased conservation between taxa and provide an evolutionarily stable set of pre-wired regulatory loci (Lewis et al. 2016; Fish, Chen, and Capra 2017). The biological context sufficient for the evolution of enhancer pleiotropy thus appears frequent enough to be found in meta-analyses of CREs. But does this context result in actual enhancer pleiotropy? The recent discovery of pleiotropic enhancers associated with development of leg bristles and trichome patterning explicitly demonstrates that pleiotropic CREs targeting developmental genes can and do underlie important traits in Drosophila (Nagy et al. 2018; Preger-Ben Noon et al. 2018).

The second principle guiding the prediction of modular CRE transfer is that recombination of extant CRE modules is a logical mechanism for the rapid introduction of new alleles into a population while mitigating deleterious effects likely to occur with coding sequence variation (Prud'homme, Gompel, and Carroll 2007; Wallbank et al. 2016). While this is undoubtedly true, past studies suggest that transfer of modular elements should not necessarily be the default assumption. In many cases, such as loss of stickleback spines (Chan et al. 2010) and horizontal stripes in cichlids (Kratochwil et al. 2018), adaptive trait evolution is driven by loss of function mutations when an organism is exposed to a novel environment. Adaptive loss of phenotype requires no assumptions regarding the modularity of a trait, as a simple deletion can be sufficient to break the regulatory architecture that underlays trait development in both modular and nonmodular scenarios (Prud'homme, Gompel, and Carroll 2007). In the gain of function case, numerous studies have highlighted the relatively rapid rate of cis-regulatory evolution (e.g. Villar et al. 2015; Lewis et al. 2016). Multiple studies have also shown that mutations within enhancer elements drive variation in complex phenotypes (e.g. Gompel et al. 2005; Nagy et al. 2018). Thus, recombination of existing CREs is certainly not required for rapid evolutionary change. Conditional on the number of CREs, distance between loci, and strength of selection against partial recombinants, evolution via directional selection or some alternate process may potentially be faster than precise exchange of multiple CRE modules.

Evidence of pleiotropic enhancers and non-modular evolution of novel phenotypes does not reject the transfer of modular CREs. Instead, these counterexamples and arguments suggest we need 
additional studies to explicitly test for adaptation via exchange of putatively modular elements. Fortunately, recent work on the evolution of mimicry phenotypes in Heliconius provides the perfect case study for how evolution of non-modular genetic architectures may drive variation in apparently modular traits.

\section{Heliconius wing patterns as a case study in non-modular phenotype evolution}

Heliconius wing patterns have been proposed as a key example of how modular cis-regulatory alleles can generate novel color pattern adaptations (Mallet and Clarke 1989; Wallbank et al. 2016; Van Belleghem et al. 2017). In Heliconius erato, red wing pattern phenotypes appear to be shuffled between hybridizing populations to produce morphs with various combinations of these pattern components. The entire list of hybrid zones described in Van Belleghem at al. 2017 is extensive, so we focus here on a particularly clear example of apparent modularity.

In French Guiana and Suriname, three red pattern morphs - named radiate, dennis, and postman (Figure 1A) - form a complex hybrid zone. Dennis, which has the forewing pattern of the radiate morph and the hindwing pattern of the postman, appears by eye to be a simple shuffling of these two other phenotypes via recombination of forewing and hindwing pattern-associated alleles. Consistent with this, DNA sequence analysis of these morphs has shown that a single locus downstream of the red "switch" gene optix is the only site that differentiates between the radiate and dennis morphs (Van Belleghem et al. 2017) (Figure 1B). When adding the postman phenotype to this comparison, we see that the locus differentiating dennis from radiate morphs contains the postman allele, rather than the radiate allele, and thus appears to be an obvious example of modular CRE transfer to create a novel phenotype.

Recent findings, however, suggest that the origin and subsequent evolution of the radiate morph is much more complicated than expected from a few simple modular CREs (Lewis et al. 2019). These results show that at least five distinct $c i s$-regulatory loci drive adaptive evolution of the radiate mimicry phenotype. Consistent with cell lineage-derived CRE availability, wing CREs do not differ between forewings and hindwings and thus the same CRE landscape is shared by both forewing and hindwing patterns (Lewis and Reed 2018). Inconsistent with modularity, pattern associated CREs are all interdependent and pleiotropic - CRE mutants alter both the rays and the dennis components of the radiate morph (Figure 2A). While hybridization seems to have spread the radiate phenotype between multiple geographic populations, recombination of a single CRE is insufficient to produce a partial or complete radiate phenotype in a postman-like morph. Perhaps most surprisingly, deletion of one CRE showed the effects of both enhancer (phenotype suppression in the mutant) and silencer (phenotype activation in the mutant) activity in different wing sections. This suggests that epistatic effects, and thus the genetic background, can play an important part in how traits evolve. This is consistent with evidence from Drosophila that activating or repressing behavior from CREs can be context dependent (Gisselbrecht et al. 2019) and recent observations in various Heliconius species that a single gene can modulate phenotype expression associated with other unlinked color pattern loci (Concha et al. 2019).

Taken together, pleiotropic CRE activity and indication of epistasis between color pattern loci firmly rejects the developmental modularity of red color pattern CREs in Heliconius erato. These 
experiments can, specifically, reject the hypothesis that a single locus is sufficient to induce wing pattern components in the absence of additional CRE alleles and the necessary genetic background. This work does, however, raise the question: Why do specific loci appear to control wing phenotypes in a modular fashion in genome sequence comparisons?

A Pleiotropic CRE interactions

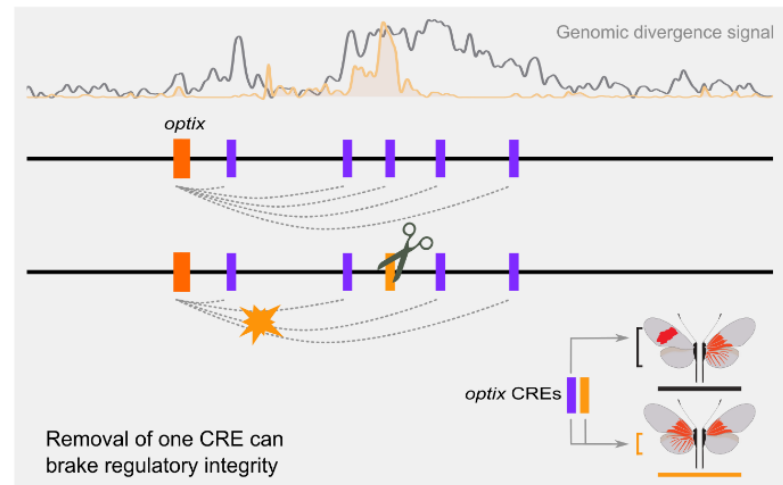

B CREs bind tissue specific factors

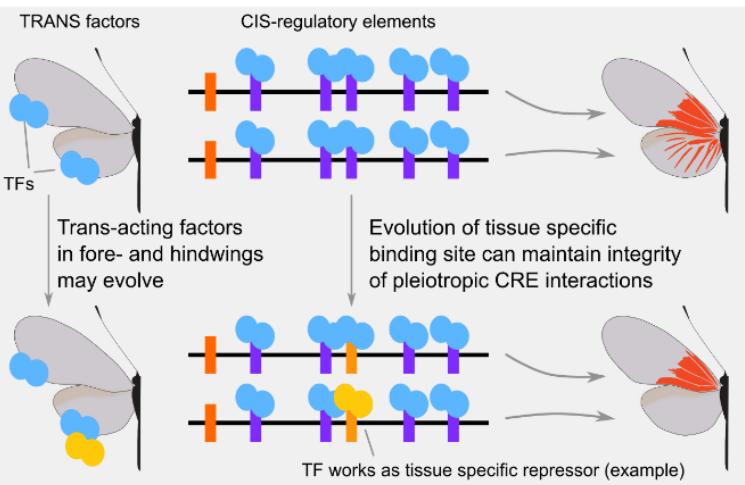

C Evolutionary modules differentiate in a homogenized background

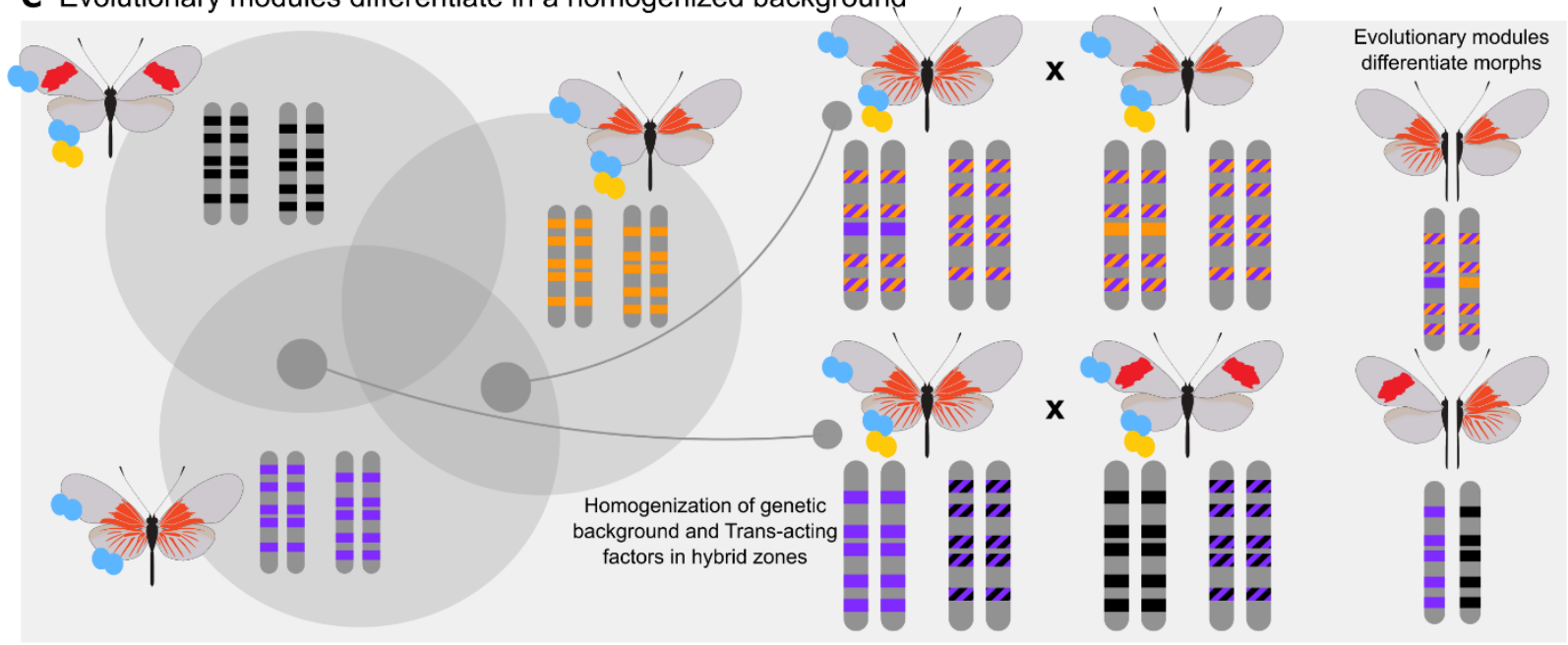

Figure 2. Conceptual overview of evolutionary modularity. (A) Red wing patterns in Heliconius erato are controlled by an interdependent set of enhancers. Loss of any enhancer causes loss of red pattern phenotype. (B) Hypothetical mechanism by which a novel, tissue specific phenotype (Dennis) might evolve through the acquisition of a tissue specific transcription factor and binding site that maintains the integrity of CRE interactions and despite CRE pleiotropy. (C) Depiction of hypothesized evolutionary modularity in the East Amazon H. erato hybrid zone. Multiple unlinked alleles control divergence between all three morphs (Left). These loci become homogenized in narrow regions of hybridization, allowing a single locus to modulate wing pattern phenotype (Right). These homogenized loci can include trans-acting factors, which will not show any signal of differentiation in genomic hybrid zone comparisons.

\section{Evolutionary modularity: a hybrid zone model}

It is important to reconcile the apparent conflict between the genomic sequence comparisons and experimental data in our case study. It is our view that comparative genomic analyses indicating modular transfer of phenotype components capture an important aspect of adaptive evolution and phenotype stability in the face of gene flow. While this approach does not demonstrate 
developmental modularity, it can provide strong evidence for a similar concept-evolutionary modularity. By evolutionary modularity, we mean: Any locus sufficient to modulate the gain or loss of phenotype components in the local genetic context of two or more hybridizing populations. This concept does not make any assumptions about the true developmental genetic architecture of a trait, but instead suggests that many architectures can be utilized in a modular fashion by evolutionary processes.

To parse out how evolutionary modularity would work, we return to our example of the East Amazon Heliconius hybrid zone. In the admixed genetic background of the hybrid zone, many combinations of pleiotropic and epistatic loci are likely to occur due to hybridization of "pure" parental phenotypes and recombination in hybrid and backcrossed offspring. When most genetic elements for a trait are homogenous among all three morphs, a single, variable locus may be sufficient to create a novel phenotype and modulate between wing pattern morphs. The apparent gain of a modular phenotype in the dennis morph can be explained as the product of this scenario: In the admixed genetic background of the hybrid zone, trans-acting factors are shared by both morphs and a single, cis-regulatory domain provides a module-like switch for swapping between phenotypes (Figure 2B,C). This single locus may be sufficient to maintain differentiation between the derived dennis morph and the radiate population, while more complicated differentiation patterns would separate radiate from postman. Thus, a single locus, insufficient for producing a phenotype in the absence of a specific genetic context, may act modular in localized population structures.

The concept of evolutionary modularity points to an important feature of adaptive evolution easily overlooked in largescale analyses of widespread populations: Evolutionary novelty arises at a specific time and place. The process of refining or separating phenotype components in derived taxa can be distinct from the processes that generate the ancestral form. This, in turn, suggests that individual hybrid zones-where modular phenotypes appear most likely — can be a breeding ground for novel organismal phenotypes via evolutionary modularity from either modular or nonmodular developmental landscapes.

\section{Facing the future for studies of adaptive phenotype evolution}

The evidence presented for non-modular evolution of red mimicry wing patterns in Heliconius provides only a single case study of whether modular CREs drive adaptation of novel phenotypes. Many more studies will be necessary before we can begin to parse the relative significance of modular and non-modular genetic architectures for phenotypic novelty and diversification. Importantly, we are not suggesting that modular genetic elements cannot or do not underlie novel phenotypes. Our perspective simply suggests that developmental modularity should not be the default assumption, even in cases where discrete phenotypes are swapped between hybridizing populations. It will be important that future cases of putative developmental modularity be demonstrated with empirical assays, rather than assumed from sequence comparisons. We also suspect, though only time will tell, that evolutionary modularity will be an important process in the production of novel phenotypes. As a deeper understanding of the genetic basis of adaptive evolution emerges, we anticipate that complex developmental architectures will repeatedly be processed in fairly simple evolutionary scenarios via hybridization and recombination to produce ecologically significant phenotypes. 


\section{References}

Campagna, Leonardo, Márcio Repenning, Luís Fábio Silveira, Carla Suertegaray Fontana, Pablo L Tubaro, and Irby J Lovette. 2017. "Repeated divergent selection on pigmentation genes in a rapid finch radiation." Science Advances 3 (5): e1602404. https://doi.org/10.1126/sciadv.1602404.

Carroll, Sean B. 2008. "Evo-devo and an expanding evolutionary synthesis: a genetic theory of morphological evolution.” Cell 134 (1): 25-36. https://doi.org/10.1016/j.cell.2008.06.030.

Chan, Yingguang Frank, Melissa E Marks, Felicity C Jones, Guadalupe Villarreal, Michael D Shapiro, Shannon D Brady, Audrey M Southwick, et al. 2010. "Adaptive evolution of pelvic reduction in sticklebacks by recurrent deletion of a pitxl enhancer." Science (New York, N.Y.) 327 (5963): 302-5. https://doi.org/10.1126/science.1182213.

Concha, Carolina, Richard W R Wallbank, Joseph J Hanly, Jennifer Fenner, Luca Livraghi, Edgardo Santiago Rivera, Daniel F Paulo, et al. 2019. "Interplay between developmental flexibility and determinism in the evolution of mimetic Heliconius wing patterns." Current Biology 29 (23): 3996-4009.e4. https://doi.org/https://doi.org/10.1016/j.cub.2019.10.010.

Fish, Alexandra, Ling Chen, and John A Capra. 2017. "Gene regulatory enhancers with evolutionarily conserved activity are more pleiotropic than those with species-specific activity." Genome Biology and Evolution 9 (10): 2615-25. https://doi.org/10.1093/gbe/evx194.

Gisselbrecht, Stephen S, Alexandre Palagi, Jesse V Kurland, Julia M Rogers, Hakan Ozadam, Ye Zhan, Job Dekker, and Martha L Bulyk. 2019. "Transcriptional silencers in Drosophila serve a dual role as transcriptional enhancers in alternate cellular contexts." Molecular Cell, December. https://doi.org/10.1016/j.molcel.2019.10.004.

Gompel, Nicolas, Benjamin Prud'homme, Patricia J Wittkopp, Victoria A Kassner, and Sean B Carroll. 2005. "Chance caught on the wing: cis-regulatory evolution and the origin of pigment patterns in Drosophila." Nature 433 (7025): 481-87. https://doi.org/10.1038/nature03235.

Kratochwil, Claudius F, Yipeng Liang, Jan Gerwin, Joost M Woltering, Sabine Urban, Frederico Henning, Gonzalo Machado-Schiaffino, C Darrin Hulsey, and Axel Meyer. 2018. "Agoutirelated peptide 2 facilitates convergent evolution of stripe patterns across cichlid fish radiations." Science (New York, N.Y.) 362 (6413): 457-60. https://doi.org/10.1126/science.aao6809.

Kuratani, Shigeru. 2009. "Modularity, Comparative embryology and evo-devo: developmental dissection of evolving body plans." Developmental Biology 332 (1): 61-69. https://doi.org/https://doi.org/10.1016/j.ydbio.2009.05.564.

Lewis, James J., Karin R.L. van der Burg, Anyi Mazo-Vargas, and Robert D. Reed. 2016. "ChIP-seq-annotated Heliconius erato genome highlights patterns of cis-regulatory evolution in lepidoptera." Cell Reports 16 (11): 2855-63. https://doi.org/10.1016/j.celrep.2016.08.042.

Lewis, James J, Rachel C Geltman, Patrick C Pollak, Kathleen E Rondem, Steven M Van Belleghem, Melissa J Hubisz, Paul R Munn, et al. 2019. "Parallel evolution of ancient, pleiotropic enhancers underlies butterfly wing pattern mimicry." Proceedings of the National Academy of Sciences 116 (48): 24174 LP-24183. https://doi.org/10.1073/pnas.1907068116.

Lewis, James J, and Robert D Reed. 2018. “Genome-wide regulatory adaptation shapes 
population-level genomic landscapes in Heliconius." Molecular Biology and Evolution, https://doi.org/10.1093/molbev/msy209.

Maan, Martine E, and Kristina M Sefc. 2013. "Colour variation in cichlid fish: developmental mechanisms, selective pressures and evolutionary consequences." Seminars in Cell \& Developmental Biology 24 (6-7): 516-28. https://doi.org/10.1016/j.semcdb.2013.05.003.

Mallet, James, and Cyril Clarke. 1989. "The genetics of warning colour in peruvian hybrid zones of Heliconius erato and H. melpomene." Proceedings of the Royal Society of London. B. Biological Sciences 236 (1283): 163-85. https://doi.org/10.1098/rspb.1989.0019.

Manceau, Marie, Vera S Domingues, Ricardo Mallarino, and Hopi E Hoekstra. 2011. "The developmental role of agouti in color pattern evolution." Science 331 (6020): 1062-65. https://doi.org/10.1126/science.1200684.

Martin, Arnaud, Riccardo Papa, Nicola J Nadeau, Ryan I Hill, Brian A Counterman, Georg Halder, Chris D Jiggins, et al. 2012. "Diversification of complex butterfly wing patterns by repeated regulatory evolution of a wnt ligand." Proceedings of the National Academy of Sciences of the United States of America 109 (31): 12632-37. https://doi.org/10.1073/pnas.1204800109.

Monteiro, Antónia, and Ondrej Podlaha. 2009. "Wings, horns, and butterfly eyespots: how do complex traits evolve?” PLOS Biology 7 (2): e1000037. https://doi.org/10.1371/journal.pbio.1000037.

Nadeau, Nicola J., Carolina Pardo-Diaz, Annabel Whibley, Megan A. Supple, Suzanne V. Saenko, Richard W. R. Wallbank, Grace C. Wu, et al. 2016. "The gene cortex controls mimicry and crypsis in butterflies and moths." Nature 534 (7605): 106-10. https://doi.org/10.1038/nature17961.

Nagy, Olga, Isabelle Nuez, Rosina Savisaar, Alexandre E Peluffo, Amir Yassin, Michael Lang, David L Stern, Daniel R Matute, Jean R David, and Virginie Courtier-Orgogozo. 2018. "Correlated evolution of two copulatory organs via a single cis-regulatory nucleotide change." Current Biology 28 (21): 3450-3457.e13. https://doi.org/10.1016/j.cub.2018.08.047.

Preger-Ben Noon, Ella, Gonzalo Sabarís, Daniela M Ortiz, Jonathan Sager, Anna Liebowitz, David L Stern, and Nicolás Frankel. 2018. "Comprehensive analysis of a cis-regulatory region reveals pleiotropy in enhancer function." Cell Reports 22 (11): 3021-31. https://doi.org/10.1016/j.celrep.2018.02.073.

Prud'homme, Benjamin, Nicolas Gompel, and Sean B Carroll. 2007. "Emerging principles of regulatory evolution." Proceedings of the National Academy of Sciences of the United States of America 104 Suppl 1 (May): 8605-12. https://doi.org/10.1073/pnas.0700488104.

Rebeiz, Mark, John E Pool, Victoria A Kassner, Charles F Aquadro, and Sean B Carroll. 2009. "Stepwise modification of a modular enhancer underlies adaptation in a Drosophila population." Science (New York, N.Y.) 326 (5960): 1663-67. https://doi.org/10.1126/science.1178357.

Reed, R. D., R. Papa, A. Martin, H. M. Hines, B. A. Counterman, C. Pardo-Diaz, C. D. Jiggins, et al. 2011. "Optix drives the repeated convergent evolution of butterfly wing pattern mimicry." Science 333 (6046): 1137-41. https://doi.org/10.1126/science.1208227.

Sabarís, Gonzalo, Ian Laiker, Ella Preger-Ben Noon, and Nicolás Frankel. 2019. "Actors with multiple roles: pleiotropic enhancers and the paradigm of enhancer modularity." Trends in Genetics 35 (6): 423-33. https://doi.org/10.1016/j.tig.2019.03.006.

Salis, Pauline, Natacha Roux, Olivier Soulat, David Lecchini, Vincent Laudet, and Bruno 
Frédérich. 2018. "Ontogenetic and phylogenetic simplification during white stripe evolution in clownfishes.” BMC Biology 16 (1): 90. https://doi.org/10.1186/s12915-018-0559-7.

Steiner, Cynthia C, Jesse N Weber, and Hopi E Hoekstra. 2007. "Adaptive variation in beach mice produced by two interacting pigmentation genes.” PLoS Biology 5 (9): e219-e219. https://doi.org/10.1371/journal.pbio.0050219.

Stergachis, Andrew B., Shane Neph, Alex Reynolds, Richard Humbert, Brady Miller, Sharon L. Paige, Benjamin Vernot, et al. 2013. "Developmental fate and cellular maturity encoded in human regulatory dna landscapes." Cell 154 (4): 888-903. https://doi.org/10.1016/j.cell.2013.07.020.

Tian, Li, Sarthok Rasique Rahman, Briana D Ezray, Luca Franzini, James P Strange, Patrick Lhomme, and Heather M Hines. 2019. "A homeotic shift late in development drives mimetic color variation in a bumble bee." Proceedings of the National Academy of Sciences 116 (24): 11857 LP-11865. https://doi.org/10.1073/pnas.1900365116.

Toews, David P.L., Scott A. Taylor, Rachel Vallender, Alan Brelsford, Bronwyn G. Butcher, Philipp W. Messer, and Irby J. Lovette. 2016. "Plumage genes and little else distinguish the genomes of hybridizing warblers." Current Biology 26 (17): 2313-18. https://doi.org/https://doi.org/10.1016/j.cub.2016.06.034.

Van Belleghem, Steven M., Pasi Rastas, Alexie Papanicolaou, Simon H. Martin, Carlos F. Arias, Megan A. Supple, Joseph J. Hanly, et al. 2017. "Complex modular architecture around a simple toolkit of wing pattern genes.” Nature Ecology \& Evolution 1 (3): 0052. https://doi.org/10.1038/s41559-016-0052.

Villar, Diego, Camille Berthelot, Sarah Aldridge, Tim F Rayner, Margus Lukk, Miguel Pignatelli, Thomas J Park, et al. 2015. "Enhancer evolution across 20 mammalian species." Cell 160 (3): 554-66. https://doi.org/10.1016/j.cell.2015.01.006.

Wagner, Günter P, Mihaela Pavlicev, and James M Cheverud. 2007. "The road to modularity." Nature Reviews Genetics 8 (12): 921-31. https://doi.org/10.1038/nrg2267.

Wallbank, Richard W. R., Simon W. Baxter, Carolina Pardo-Diaz, Joseph J. Hanly, Simon H. Martin, James Mallet, Kanchon K. Dasmahapatra, et al. 2016. "Evolutionary novelty in a butterfly wing pattern through enhancer shuffling." PLOS Biology 14 (1): e1002353. https://doi.org/10.1371/journal.pbio.1002353.

Westerman, Erica L, Nicholas W VanKuren, Darli Massardo, Ayşe Tenger-Trolander, Wei Zhang, Ryan I Hill, Michael Perry, et al. 2018. "Aristaless controls butterfly wing color variation used in mimicry and mate choice." Current Biology : CB 28 (21): 3469-3474.e4. https://doi.org/10.1016/j.cub.2018.08.051.

\section{Acknowledgments}

We thank Leo Campagna and Vincent Laudet for providing us with images for capuchino seedeaters and clownfish, and Karin van der Burg for helpful comments.

\section{Authors contribution statement}

JJL and SMVB contributed equally to the presented ideas, writing of the manuscript and making the figures. 


\section{Funding}

2

3 JJL was supported by NASA 17-EXO-17-2-0112 and NSF DEB-1546049. SMVB was supported 4 by NSF EPSCoR RII Track-2 FEC (OIA 1736026) and in part by National Institutes of Health-

5 NIGMS COBRE Phase 2 Award - Center for Neuroplasticity at the University of Puerto Rico 6 (Grant No. 1P20GM103642). The content is solely the responsibility of the authors and does not 7 necessarily represent the official views of the National Institutes of Health. 